INTERNATIONAL DESIGN CONFERENCE - DESIGN 2018

https://doi.org/10.21278/idc.2018.0188

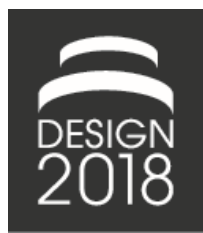

\title{
AN APPROACH FOR THE IMPLEMENTATION OF THE DIGITAL TWIN IN THE AUTOMOTIVE WIRING HARNESS FIELD
}

\author{
R. Tharma, R. Winter and M. Eigner
}

\begin{abstract}
The importance of continuous and sustainable information exchange processes rises, due to the growing digitalisation in many fields of automotive area. By implementing the Digital Twin method, these challenges can be met in the future. In this paper, the Digital Twin method is discussed in the context of the automotive wiring harness. Different kinds of individual definitions of the Digital Twin are discussed and the methodical meaning is analysed. Furthermore the wiring harness specific requirements for the Digital Twin, caused by the enormous variety, is considered.
\end{abstract}

Keywords: digital twin, wiring harness, complexity, configuration management, data driven design

\section{Introduction}

In times of increasing digitalisation and continuous transformation of the automotive markets it is essential to adapt to this increasing change by way of development, production, and service processes of a product. One of the most critical tasks, which has to be considered is making the operations during the whole lifecycle of a product as efficient and intelligent as possible. In order to do this, sustainable information exchanges between the individual stakeholders have to be created. These requirements fuel the use of complex virtual product models, which are commonly referred to as Digital Twins.

Based on these Digital Twins of a physical product it will become possible to make efficient predictions of how changes in product, process development, and a new kind of operating and service workflow, will affect the product performance. (Schleich et al., 2017). Driven by current information technology, the integration of electrical and mechanical components require the Digital Twins to be able to generate consistent and exhaustive documentation of new kinds of products and services (Gruender, 2017). Due to the actual lack of a concrete case of the application for Digital Twins this contribution introduces a concept for implementation of the Digital Twin method within the scope of the wiring harness development. The main questions, which are addressed within this contribution are: What are the challenges in current wiring harness development? Which requirements are needed for a Digital Twin? How to handle a large amount of product variance and still make a $100 \%$ accurate and complete wiring harness? What are possible use cases for the individual stakeholders?

\section{State of the art in the wiring harness field}

Driven by the vast amount of rules and criteria, which have to be obeyed during the development of new kind of electrical and electronic architectures, wiring harness development is getting more challenging than ever (Beck et al., 2016). The following chapters contain information regarding the actual 
complexity of the wiring harness for passenger cars, moreover it is shown what kind of new methods are used to meet these challenges.

\subsection{Automotive wiring harness development}

The wiring harness in vehicles is responsible for connecting all the hardware components, such as control units and any electronic parts. The wiring harness is the central nervous system of each car. Besides this vast importance there are many barriers during the development process. Starting with the challenging chronological sequence, the wiring harness is dependent on the maturity of the body-inwhite and the positioning of the electronic devices. Thus it should be necessary that the wiring harness be nearly one of the last parts designed.

In reverse order the wiring harness is one of the first parts built in the in the body-in-white at the assembly line. Furthermore, the physical characteristic of the flexible wiring harness leads to design difficulties. To resolve these issues a Digital-Mock-Up (DMU) is used during the design processes. The DMU represents the wiring harness in a close to reality volume of data, which serves as a basis for the virtual product development and enables the verification of installation investigations in the very early stages of vehicle development.

This kind of virtual wiring harness development allows integrating the suppliers in the processes for creating a parallel and embedded development process for close coordination (Eigner et al., 2011).

Further issues, which fuel the rising complexity and wide variety of existing wiring harnesses for each vehicle are, on the one hand, dependant on the vehicle configuration, which is based on the customer wishes. To clarify the dimensions of possible configurations for a middle-class Mercedes-Benz passenger car, a total amount of about $10^{27}$ can be named (Zagel, 2006). Depending on the configuration, the installed vehicle functions and electronic components that have to be connected from the wiring harness, vary. On the other hand, the continuous cost savings from these practices lead to the development and building of a customer-specific wiring harness. This contains as few waste wires and waste parts as possible that would not be connected due to the customer ordered vehicle configuration (Bortolazzi et al., 2004). What this means for wiring harness development is that it needs to provide the most convenient solution to a wide variety of situations and at the same time handle a massive amount of product data. Therefore, the vehicle is separated into assembly spaces that are defined by considering all possible production scopes from the supplier on one formboard at once. These assembly spaces can be differentiated by the amount of waste wires and parts that at their worst could be in individual spaces. For example, in the interior there are a lot of customer-specific options selectable, which means there are also a lot of electronic components that can be ordered and built in or not.

It makes sense to develop a customer-specific wiring harness for the interior to avoid waste of unneeded cable and parts. Conversely, in the door section there are not as many possibilities to configure making this a classic case for a non-customer-specific wiring harness. In this case, as few as only one wiring harness is developed and designed for all possible vehicle configurations with all possible connecting possibilities for each kind of seat type. The sectioning of the vehicle in non-/ and customer-specific wiring harness areas is shown in Figure 1.

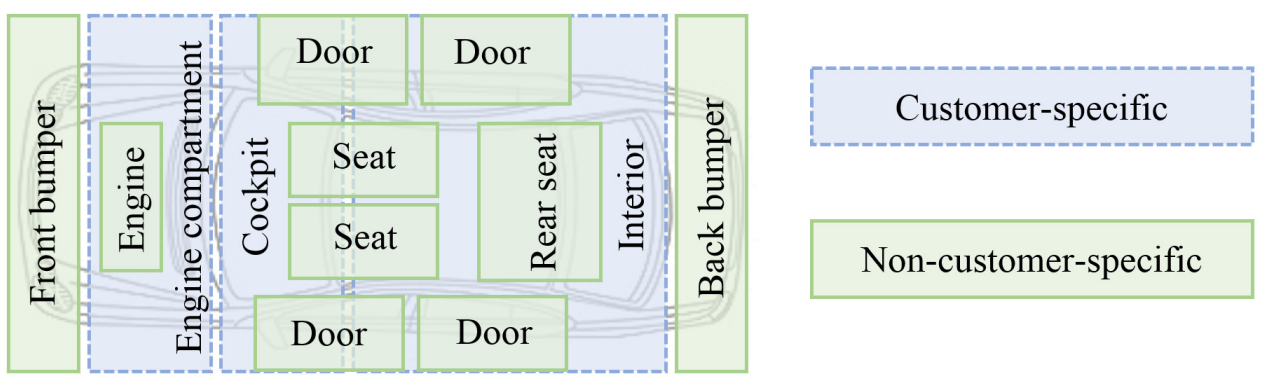

Figure 1. Vehicle sectioning in physical wiring-harness 


\subsection{Usage of 3D-Master models}

The 3D-Master method, which follows the intention to design and develop products without drawings, is a new way to develop the wiring harness. The advantage of using this method is the possibility to derive all information for the receptive product directly from the 3D-Master model.

In the case of the wiring harness development, the 3D-Master Method combines the assembly spaceoriented design on the one hand, and the hardware-oriented design on the other hand (Neckenich et al., 2015). This ensures that all the necessary information about the wiring harness is directly visible in the digital data model. For each shown assembly space in Figure 1, there will be a unique digital data model in the end.

Especially important to notice at this point is, that the digital data model is not created for each vehicle derivate. But it is designed for each steering type of a vehicle series and covers at the same time as many derivates as possible, which is dependent on the needful similarity in topology. That is why it is called the master model. Another condition for defining the master is that all of its wiring harness modules together can be manufactured on one single formboard (Neckenich et al., 2016).

The master of each assembly space contains wiring harness modules from several derivatives of the respective steering type of a vehicle series. Digital data models based on this master contain the maximal wiring harness product information for each assembly space, this is also called " $150 \%$ information" (Bogner et al., 1998).

Such a " $150 \%$ wiring harness", like in any master is not even available for order from the customer. The necessary information for this exhaustive product definition is based on the flow of information between the wiring harness database, wiring diagram, modularisation and the assembly space model (Neckenich et al., 2016). The database contains several pieces of data and information about the usability application standards and manages the relations between the diverse elements.

The wiring diagram provides the logical information for the wiring harness. It contains the necessary circuitry and references for the electronic relations between the diverse components. In the modularisation process, the whole wiring harness of an assembly-space is getting separated into single modules. These modules usually mirror the functions, which are available for order from the customer. The assembly space models are static objects of the installation position in the vehicle, which represent the maximal need of assembly space.

In addition to these geometrical objects, there is more space information implemented in the models, which are necessary due to manufacturing reasons or future innovations. The Output of the 3D-Master Model is a data container, which contains a "Harness Description List" (KBL) (Neckenich et al., 2016). The KBL was recommended by the VDA 4964 in 2005 as a file format for exchange harness design data within OEMs, suppliers and manufactures (ProSTEP iViP, 2009). A uniform file format enables standardized exchange processes, viewing solutions and references for better data quality and avoids repeated development of engineering data (VDA, 2014).

As an output of the 3D-Master Model, there is also a 3D-model container, which is necessary for geometrical specification and description of the parts. With all these relevant information for the " $150 \%$ digital data model", the master model data container is used for documentation and release processes of the respective wiring harness.

\section{The Digital Twin}

The first time, when a "Twin" of a physical entity was created, dates from several years ago to NASA's Apollo program. There were built at least two identical space crafts for mirroring the condition of the one spacecraft, which is currently in space with the one on earth - the craft on the earth was called "the twin" (Boschert and Rosen, 2016).

Since then until now there are several kinds of definitions have been established for the meaning scope of the "Digital Twin". For example, the automobile manufacturer "TESLA" is planning to develop a Digital Twin for each vehicle and to synchronize information exchange between the vehicle and the factory. The technology company "PTC" is aiming to create a link between the physical entity and the virtual model to enhance the production processes (Schleich et al., 2017). 
The software company "Dassault System" is focusing on product design performance. The conglomerate "General Electric" planning to create Digital Twins from their product to predict the health and performance over a lifetime (Schleich et al., 2017).

Also regarding some scientists, there are small or more significant differences in the view of the benefit and scope of the Digital Twin.

Regarding to the first scientist, who spoke about the Digital Twin "A Digital Twin is an integrated multiphysics, multiscale simulation of vehicle or system that uses the best available physical models, sensor updates, fleet history, etc., to mirror the life of its corresponding flying twin" (Shafto et al., 2010, 2012).

According to Michael Grieves, who is one of the pioneers in this task, the Digital Twin is "a set of virtual information constructs that fully describes a potential or actual physical manufactured product from the micro atomic level to the macro geometrical level" (Grieves et al., 2017).

To combine all the different definitions of a Digital Twin, it could be said, that a Digital Twin of a real distributed product is a virtual reflection, which can describe the exhaustive physical and functional properties of the product along the whole life cycle and can deliver and receive product information. The following figure shows the development process of the Digital Twin integrated into the three phases of every products life cycle, design, production and service.

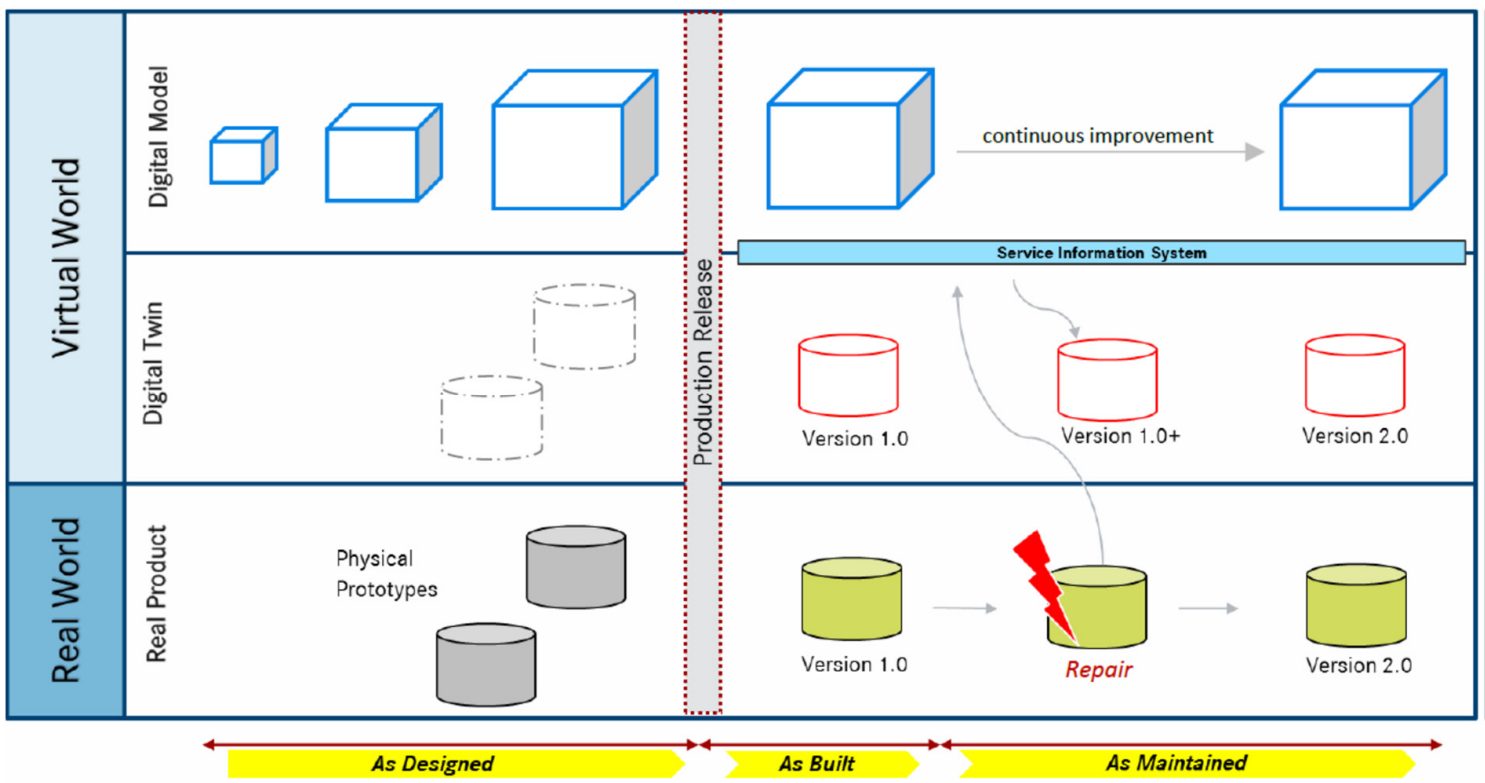

Figure 2. Visualisation of the Digital Twin development process (Eigner, 2017)

As we can see in Figure 2, a current product development process is always divided in a real and a virtual world, where the different phases of a product appear. Every development starts in the virtual world with digital models, which contain more or less the complete digital product and function description that are created and improved until they reach the product's individually defined level of maturity.

Based on this version of the digital model physical prototypes are built in the real world to do several product checks such as installation investigations, functionality tests, safety, and durability checks. The know-how, which is gained during the physical prototype tests in the real world, is always in close cooperation with the digital model in the virtual world so that the physical prototypes can be improved continuously.

When the physical prototypes have passed the whole individual product checklist for test cases and the digital model has been updated continuously to the latest version, then the design-phase of the life cycle can be considered as completed and the digital model can be released for series production.

In case of difficulties and errors during the manufacturing process, the production can fix the specific problem and do a second release for the updated digital product documentation. The birth of the Digital Twin is at the time when the manufacturing process of a product is finished, and it is ready to hand over to the customer. 
A Digital Twin meets the challenge to reduce the digital model to the only necessary information, which is needed for all the phases of a products life cycle. Another point is to cover all the data, which is required for a $100 \%$ realistic representation of the physical product, depending on the individual customer-specific configuration.

Similar to human twins in real life, who are related lifelong, the intention of a Digital Twin is to be linked with the physical product for the entire lifetime. Therefore, the Digital Twin has to be implemented in a central data layer or an IoT-Platform from which the physical entity and the Digital Twin can exchange information, for example about the parts which got damaged and had to be a repaired (Figure 2). In this case, the workshop would send a report to their service information system, where all the information about the done repair is documented. This service information system should provide this information to the data layer or IoT-Platform, where the Digital Twin can be updated automatically (Eigner, 2017). This way, it can be ensured that the database of the Digital Twin is the realistic representation of the physical entity along the entire lifetime.

\subsection{Distinction between Service Twin and Production Twin}

In the detailed formation process of a Digital Twin three kinds of phases have to be considered to define various scopes of digital product information. As mentioned in the chapter before, the basis for creating a Digital Twin is the complete and mature digital model, which results from the development and design phase. This digital model is the first unit in the Digital Twin process and represents the product release, which is based on the " $150 \%$ bill of materials" and includes all relevant product variants.

Depending on the customer-specific order, the digital model has to be reduced from the " $150 \%$ digital product description" to the $100 \%$ realistic representation that is called "Production Twin" (Eigner, 2017). This twin contains all the complete information about how the product is getting built during the production. If the physical product is a vehicle, this Production Twin would contain the information about all parts, from the body-in-white, over the engine and until the tires.

Product-dependent it has to be determined if it is possible and necessary to trace all the implemented parts in the respective product. To remain with the example of the vehicle, it is impossible nowadays to be able to trace the kind of tires, which the owner has mounted, because he has the choice to go to a workshop and mount the tiers or to do it by himself. Also, he has the choice to buy new tires or take some from a second-hand dealer. The same case can be adapted to the wiper blades, light bulbs or fuses. Simplified it can be said, that the tracing of parts that are interchangeable is not possible or rather wise. So the Production Twin contains in some cases of products unnecessary digital product information, which can be disregarded. A Digital Twin without that nonrelevant information is called "Service Twin" that contains parts, which are traceable by the service information system (Eigner, 2017). This context is visualised in the following figure.
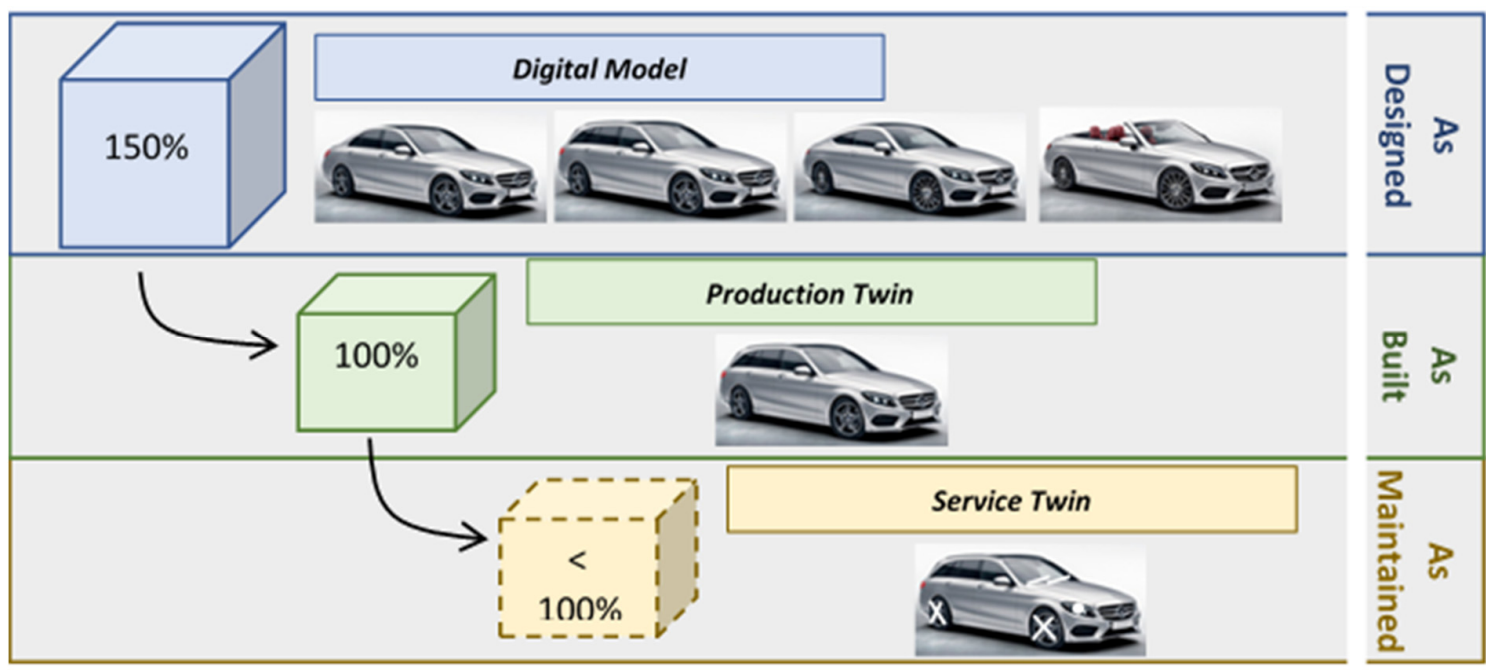

Figure 3. Distinction between digital model, Production and Service Twin 


\subsection{Other to be considered aspects in the Digital Twin process}

The prerequisite for creating a sustainable and intelligent Digital Twin process is to discuss points, which focus on fundamental questions that are listed in the following. It has to be thought about those aspects, which are similar for all kinds of physical products that should have a lifelong digital twin.

\subsubsection{Composition}

The modern product development processes are all based on PDM or PLM systems that can break down the composition of a product in a structured method. This structured composition allows the user to trace, which part or version is the predecessor or rather the successor of the actual component. The respective component family is also visible for example, a side mirror belongs to the family doors, and a heads-up display belongs to the family cockpit.

In the context of the Digital Twin the question needs to be answered whether it is necessary to define the composition of a production or rather a Service Twin in that structured method, or is a simplified flat composition sufficient. In a "flat composition" there are not any component families or rather predecessor or successor for a component, but this composition contains only the master information about the respective parts.

If any use cases require more information, the respective part and the additional information can be determined in the central PDM or PLM system, by using the master data.

\subsubsection{Enrichment of additional information}

The Digital Twin is a method that provides relevant information for all the stakeholders along the whole life cycle of a product. The " $150 \%$ digital data model" has to be validated if all the necessary information, which is essential or required for further use cases of the stakeholder, are existing. Therefore, a more or less extensive research depending on the type of product should be done with the stakeholder that are involved in the life cycle.

\subsubsection{Storage of the Digital Twin}

To enable the sustainable and continuous information exchange between the physical entity and the Digital Twin, the aspect should be considered, where the Digital Twin should be stored after birth. Nowadays improving technologies become an excellent base for the use as a Digital Twin homeland. One of the examples that are for sure a possible solution for this question is an enterprise IoT platform, which enables to link directly to the physical product. Another solution may be to create an overarching data layer within the organisation by considering the several stakeholders during the life cycle, where also the relevant digital data information can be exchanged.

\subsubsection{Reduction of the digital model to a Digital Twin}

For creating a comprehensive digital representation of a physical product, the digital model should be reduced in a thought out method. Therefore the content of the digital model has to be validated and it has to be determined if the data is available lifelong. Furthermore, the consistency of the data quality should be checked, which should be defined as a standard in the best case.

Finally, there should be thought about, how the " $150 \%$ information volume" of a digital model that covers data for several product variants, can be reduced into a 100\% Digital Twin that represents the respective one product variant.

\section{Digital Twin in context of the wiring harness}

As already mentioned in the chapters before, the wiring harness of a vehicle is not only a single part. It is a customer-specific configuration assembly of various modules that have their specific part identification and their subparts like connectors, plugs or any mechanic components.

In the premium car segment, it is usual to install more than 130 single modules to an assembly wiring harness. This complex structure leads to an enormous variety of data, which has to be considered. The $3 \mathrm{D}-$ Master model allows to meet this requirement, due to its content of the production released " $150 \%$ wiring harness digital model", which is the necessary breeding ground for a sustainable Digital Twin in 
the wiring harness development. Alongside this issue, the need for the information about the correct wiring harness modules that are installed in each vehicle is given. Depending on the ordered vehicle model and the consumer country, the wiring harness is produced in one of the plants across the world. These plants get supplied in some cases by their own suppliers with their own logistics and documentation processes, which leads to an increase of complexity.

When those requirements are fulfilled, methods and processes have to be created for reducing the " $150 \%$ digital model" by using the wiring harness information of a specific vehicle and to finally create a Digital Twin. Also, the methods and processes have to allow the Digital Twin to live for the whole lifetime of the physical vehicle and to adopt all the updates caused due to repair services or component failures.

As the home of the Digital Twin, a data layer should be created, which enables also interfaces to the service information system for lifelong information exchange. Based on the data layer, the different stakeholders during the lifecycle are able to upload and exchange product-specific information, by using their own role-specific interface to the Digital Twin. The following figure shows the mentioned points across the three lifecycle phases, as designed, as built and as maintained.

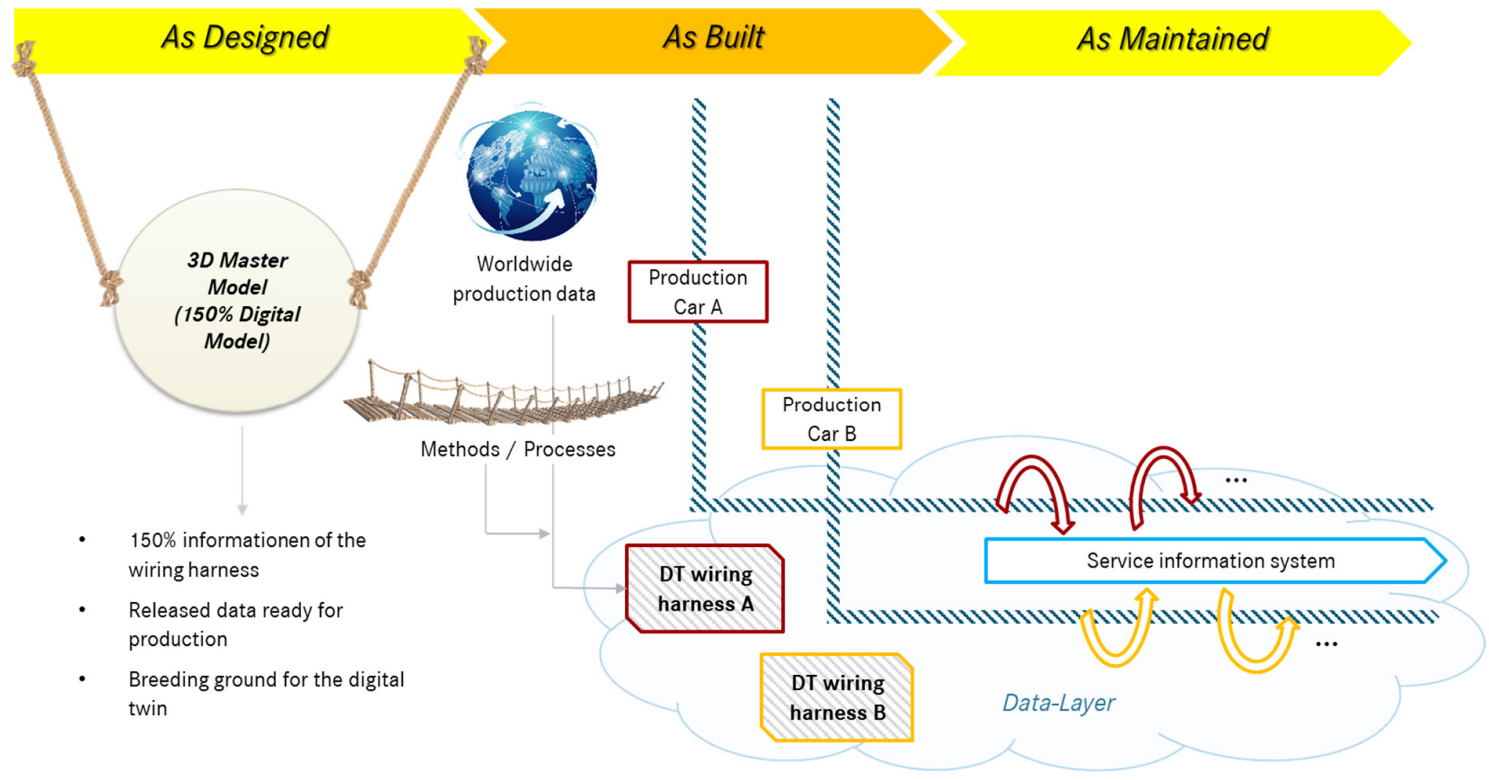

Figure 4. Digital Twin in context of the wiring harness

\subsection{Reduction of the digital model}

The key question that has to be answered before considering the reduction methods for the digital model is: Who are the users of the Digital Twin during the lifecycle? If the answer for this question is determined, the necessary content of a Digital Twin can be derived.

In case of the wiring harness issue, the primary users will be the development departments, the respective production-departments, and also the after sale departments, who are responsible for the repairs in case of wiring harness damage. Within the respective Mercedes-Benz Cars departments it can be summarised, that in the matter of the wiring harness the Digital Twin should contain a 3Dvisualisation, the wiring logic, and also the used part master data of the installed $100 \%$ wiring harness. Based on the digital model, which contains among other data a "Harness Description List" (KBL) and 3D-model container (Neckenich et al., 2016), a Digital Twin can be created. The primary source of information is the KBL, which is derived from the released 3D-master model. In addition to the detailed information about the " $150 \%$ wiring harness" the $3 \mathrm{D}$ geometrical information for the segments, which is derived from the coordinates of the splines in the CAD, are implemented in the KBL.

By using a suitable Tool, the " $150 \%$ wiring harness" that is described by the KBL can be visualised. Due to the fact, that in the KBL several connectors, clips and other parts, which are installed in each 
wiring harness module and connected with the segments, are only named by their reference, it is not possible to visualise these parts and the segments at the same time.

So by using the KBL, the segments of the " $150 \%$ wiring harness" can be shown in $3 \mathrm{D}$ with the reference of the installed parts, but not the 3D-geometry of the parts itself. Therefore, the method has to make use of the 3D-model container, which contains all 3D-geometry data with the references to every single component used in the " $150 \%$ digital model" in JT-data type.

Accordingly it is possible to combine the 3D-visualisation of the " $150 \%$ wiring harness segments", which results from the KBL and the 3D-geometry of the needed parts by using the reference identifications. As a previous step, it should be checked if the 3D-visualization of the KBL is able to be converted in a JT data type, which is the smoother solution for combining.

To reduce from the " $150 \%$ to the final $100 \%$ wiring harness that is installed in a vehicle, the respective wiring harness modules, which result from the production plant-specific system for the bill of materials have to be determined and selected. One of the challenges by creating the $100 \% 3 \mathrm{D}$-visualisation out of the digital model is to adapt the bundle diameter that is depending to the amount and kind of installed wiring harness modules, which themselves, are depending from the customer-specific vehicle configuration. Furthermore, the placement of mass positions, equipping of fuse boxes, diameters of splices, and other tasks, which are all dependant on the configuration, have to be considered. Afterwards, the master data of the used parts and wires can be implemented directly in the $100 \% 3 \mathrm{D}$-visualisation of the wiring harness. Finally the Digital Twin contains besides the JT-geometrical information of the parts, the knowledge of the $100 \% \mathrm{KBL}$, which is structured in 3D-geometrical information of the segments, the references of the connected parts, the routing information for the $100 \%$ wiring harness and some additional product manufacturing information (PMI). It should also be ensured, that a simplified visualisation of wiring diagram of the $100 \%$ wiring harness can be created on the fly by using the implemented routing information.

\subsection{Traceability between the digital model, Digital Twin and the real entity}

In case of errors during production or damages in the components of the wiring harness, the traceability between the respective physical vehicle, the Digital Twin, and also the digital model should be given. The Digital Twin links the virtual world and the physical world and should accordingly act like an adapter for tracing from the physical vehicle to the digital model and the other way around.

Every single passenger car, which is driving in the world has got its identification that is called "Vehicle Identification Number (VIN)". This Number is printed in the body-in-white during the production of the vehicle and is used for identifying the exact car along the whole lifetime. Therefore it makes sense to implement this VIN also into the respective Digital Twin, which has to be also be connected to the vehicle its entire lifetime.

On the other side, each 3D-Master model or rather each digital model has got its identification number. Due to the fact, that the Digital Twin is assembled from various digital models, like a digital model for the cockpit, digital model for the interior or digital model for the engine compartment, it is also advantageous to implement the identification number of those using digital models into the Digital Twin.

These logical links allow determining all physical vehicles, which are using wiring harness modules from a specific digital model. In reverse order, it is possible to decide on, which digital models are the origins of the physical wiring harness that is installed in a specific vehicle.

\subsection{Use cases of the Digital Twin in the wiring harness field}

In this chapter, there are mentioned some potential use cases of the Digital Twin in the wiring harness field, which considers the several stakeholders from the life cycle. The development departments can profit from the Digital Twin in case of vehicle errors due to damage in the wiring harness module.

By using the traceability it is possible to detect and solve the problem quickly and cost-efficiently. Another point is the possibility to improve the product knowledge due to the historical data, which is gained during the lifetime of a vehicle. For example, the majority of the cars have some form of electrical component damage due to grommets, which can leak in later years. Based on the gained Digital Twin 
knowledge, the importance of improving this specific grommet can be determined and the problem can be solved for future vehicles.

The production departments, which are responsible for installing the wiring harness in the vehicles can also profit from the Digital Twin. Due to the vehicle specific 100\% wiring harness data, the Digital Twin allows determining the exact amount of all the necessary plugs, which have to be plugged in during the manufacturing in the shop floor. By listing all the plugs that have to be considered for any specific wiring harness, it can be ensured, that the human-made failure rate during manufacturing will decreases. After sales departments can have many benefits by using a Digital Twin as well. For example, the mean time to repair can be decreased because the workshop employees would need less time to find the impacted components by using the 3D-visualisation of the vehicle specific wiring harness. Similar to the advantages in the development departments, the after sales departments can fall back on a vast database of reasons and solutions for several kinds of vehicle and component failure, which are documented in various Digital Twins, to create more efficient and customer friendly workflows.

Furthermore, the marketing and sales departments could also have advantages by using the Digital Twin. The possibility to visualise the customer-specific vehicle and also to calculate its configuration dependant weight, is for sure a new way of marketing and sales. Even the purchase departments could profit, due to the database with all the installed components and wires within the last years, which is created by the Digital Twins and can be used for further purchase analysis and statistics.

In the light of the new upcoming test procedure "Worldwide harmonized Light vehicles Test Procedure" (WLTP), which was developed for the determination of pollutant, fuel consumption and $\mathrm{CO}_{2}$ emissions for every single vehicle more realistic, the digital twin is getting even more important. The WLTP test procedure considers beside a new driving cycle also the $\mathrm{CO}_{2}$, which is caused by the additional weight due to optional equipment of a customer specific vehicle (Schütz, 2016). Also for those new WLTP requirements, the implementation of the Digital Twin method in the documentation processes could be useful. Based on the Digital Twins, which should be enriched with additional information, like weightand material-details, the vehicle-specific pollutant, $\mathrm{CO}_{2}$ and fuel consumption could be calculated and displayed for sales and marketing purposes.

\section{Conclusion and prospect}

The importance of information exchanges between the stakeholders of a product lifecycle is continuously increasing, which leads to the need for virtual products that describe the physical entity entirely in the virtual world.

This kind of virtual products are called Digital Twins and allow a lot of new possibilities and advantages in the future product documentation field. This work introduces the Digital Twin in context of the wiring harness found in Mercedes-Benz Cars. Among state of the art and fundamental topics to be considered, there are also given steps, which are needed for implementing a Digital Twin in the current wiring harness development process. Furthermore, the users of a Digital Twin are determined, and also their use cases and benefits are mentioned. In the future, there will be more topics, which have to be considered for realising a sustainable and convenient Digital Twin process.

One of the main topics, which has to be considered is the need of a data-layer, an IoT-platform or a cloud that enables the twins to stay continuously in touch with the respective physical entity. Furthermore, the methods and processes for creating a Digital Twin have to consider, that the Digital Twin should be prepared for archiving over a lifetime.

Another point that should be reflected is the method for 3D-visualisation of the wiring harness that is representing a product with a huge variety. The advantages of creating unique views for Digital Twins, which are dependent on the stakeholder, have to be determined. For example, the marketing departments don't need the same view of content as the development department. Finally the necessary methods for enabling a continuous information exchange between the "As Maintained"-phase and the Digital Twin have to be created and validated.

\section{References}

Beck, T., Reichmann, C. and Schaeuffele, J. (2016), "Modellbasierter Ansatz vom Architekturentwurf bis zur Serienreife", ATZ elektronik, Vol. 6, pp. 58-63. 
Bogner, R., Hoyer, P.-J., Kyriazis, J. and Urban, G. (1998), "VOBES - Das Volkswagen-Bordnetze Entwicklungssystem", Elektronik im Kraftfahrzeug, Electronic Systems for Vehicles, Tagung, Baden-Baden, Germany, October 8-9, 1998, VDI-Verlag, Düsseldorf, pp. 1085-1100.

Bortolazzi, J., Garthe, M. and Menrad, B. (2004), "Elektronik und Vernetzung”, Motortechnische Zeitschrift, Vol. 65, pp. 106-115.

Boschert, S. and Rosen, R. (2016), “Digital Twin - The Simulation Ascpect”, In: Hehenerger, P. and Bradley, D. (Eds.), Mechatronic Futures, Springer, pp. 59-74. https://doi.org/10.1007/978-319-32156-1_5

Eigner, M. (2017), "Workshop: Digital Model \& Digital Twin”, Proceedings of the ProSTEP iViP Symposium 2017, Essen, Germany, May 17-18, 2017.

Eigner, M., Gerhardt, F., Gilz, T. and Mogo Nem, F. (2011), Informationstechnologie für Ingenieure, Springer Vieweg, Kaiserslautern. https://doi.org/10.1007/978-3-642-24893-1

Grieves, M. and Vickers, J. (2017), "Digital Twin: Mitigating Unpredictable, Undesirable Emergent Behavior in Complex Systems", In: Kahlen, J., Flumerfelt, S. and Alves, A. (Eds.), Transdisciplinary Perspectives on Complex Systems, Springer. https://doi.org/10.1007/978-3-319-38756-7 4

Gruender, W.T. (2017), "Systems Engineering Requires Digital Twins of Machine Elements", CONAT 2016, International Congress of Automotive and Transport Engineering, pp. 227-233. https://doi.org/10.1007/9783-319-45447-4 26

Neckenich, J., Winter, R. and Vielhaber, M. (2015), "Digital Support of wiring harness development (Based on the 3D Master Method)", Proceedings of the ICED15 / International Conference on Engineering Design, Milano, Italy, July 27-30, 2015, pp. 1-10.

Neckenich, J., Zielbauer, U., Winter, R. and Vielhaber, M. (2016), “An integrated approach for an extended assembly-oriented design of automotive wiring harness using 3D Master Models", Proceedings of the DESIGN 2016 / International Design Conference, Durovink, Croatia, May 16 - 19, 2016, pp. 717 - 726.

ProSTEP iViP (2009), Fahrzeugelektrik, Meilensteine zum Digitalen Fahrzeugmodell, White Paper, ProSTEP.

Schleich, B., Anwer, N., Mathieu, L. and Wartzack, S. (2017), "Shaping the Digital Twin for design and production engineering", CIRP Annals - Manufacturing Technology, Vol. 66, pp. 141-144. https://doi.org/10.1016/j.cirp.2017.04.040

Schütz, T. (2016), "WLTP - On the increased importance of aerodynamics and impact on development procedures", The Proceedings of 16. Internationales Stuttgarter Symposium, Automobil- und Motorentechnik, pp. 1-17. https://doi.org/10.1007/978-3-658-13255-2_1

Shafto, M., Conroy, M., Doyle, R., Glaesgen, E., Kemp, C. et al. (2010), Draft modeling, simulation, information technology \& processing roadmap, National Aeronautics and Space Administration.

Shafto, M., Conroy, M., Doyle, R., Glaesgen, E., Kemp, C. et al. (2012), Modeling, simulation, information technology \& processing roadmap, National Aeronautics and Space Administration.

VDA (2014), VDA 4964 - Harness Descrption List, VDA.

Zagel, M. (2006), Uebergreifendes Konzept zur Strukturierung variantenreicher Produkte und Vorgehensweise zur iterativen Produktstruktur-Optimierung, $\mathrm{PhD}$ thesis, University of Kaiserslautern.

Rajeeth Tharma, PhD candidate

Daimler AG, RD/UBL

Bela-Barenyi-Str Tor 14, 71063 Sindelfingen, Germany

Email: rajeeth.tharma@daimler.com 